\title{
Inkjet Printing and Cell Seeding Thermoreversible Photocurable Gel Structures
}

\author{
Manuela Di Biase, ${ }^{a, b}$ Rachel E. Saunders, ${ }^{a}$ Nicola Tirelli, ${ }^{a, b}$ and Brian Derby ${ }^{* a}$ \\ ${ }^{\text {a }}$ School of Materials, University of Manchester, Oxford Road, Manchester, M13 \\ 9PL, UK. Fax: +44 161 3068877; Tel: +44 161 3063569; E-mail: \\ brian.derby@manchester.ac.uk \\ ${ }^{\mathrm{b}}$ Faculty of Medicine and Human Sciences, University of Manchester, Oxford Road, \\ Manchester, M13 9PL, UK.
}

We have developed a biocompatible fluid suitable for inkjet delivery that gels by a tandem mechanism of a rapid physical gelation followed by a photoactivated chemical cross-linking. We prepared $20 \%$ vol. aqueous solutions of acrylate functionalised Pluronic F127, poly(ethylene glycol-bl-propylene glycol-bl-ethylene glycol) (PEO-PPO) with triethanolamine and eosin-Y as a photocurable cross-linker; in order to minimise phase separation a small amount of polyethylene glycol diacrylate were added to the solution. This fluid has a viscosity $<20 \mathrm{mPas}$ at $5{ }^{\circ} \mathrm{C}$ and is suitable for inkjet printing. We used a piezoelectric drop-on-demand inkjet printer at this temperature to print single and multilayer structures on a substrate held at room temperature. The dimensions of the resulting structures are consistent with models developed for the interaction of overlapping drops. After photocrosslinking the resulting gels, which are stable in an aqueous environment, were successfully seeded with fibroblast cells also delivered by an inkjet printer. 


\section{Introduction}

A major obstacle to the development of tissue engineering, cell-based microreactors and biosensors is the availability of controlled and well-defined scaffold structures upon which cells adhere and spread and on which their metabolic and proliferative activity can be conveniently studied in response to external stimuli. A technical hurdle in this area is the lack of fabrication techniques for generating scaffolds, with complex internal architecture, into which cells can be deposited in a controlled manner and in environments of controlled morphology and chemistry. Here we present a study of the use of inkjet printing to directly fabricate scaffold structures from biomaterials and to co-seed cells within the scaffold as it is being fabricated.

There has already been preliminary work carried out in this to demonstrate the basic feasibility of this concept. Boland, ${ }^{1}$ Nakamura $^{2}$ and earlier work by ourselves ${ }^{3}$ has demonstrated that a range of mammalian cells can be deposited by inkjet printing and that these cells survive, adhere to substrates and grow to confluence. However, the requirements of inkjet printing restrict the range of biomaterials that can be codeposited with cells.

Inkjet printing has been recently reviewed as a materials fabrication tool ${ }^{4}$ where it is argued that the most appropriate inkjet tool for the delivery of materials is piezoelectrically actuated drop-on-demand (DOD) inkjet printing. Hence, despite Boland successfully demonstrating the use of thermal inkjet technology for cell

dispensation, ${ }^{1}$ we have chosen piezoelectrric DOD inkjet for this study. The physical properties of fluids that are compatible with drop-on-demand inkjet printing have 
been discussed in detail elsewhere and can be expressed in terms of a parameter space with axes of the Reynolds and Weber numbers (Re and We respectively). ${ }^{4,5}$ The fluid conditions suitable for printing have been identified through a combination of dimensional analysis and numerical simulation 6 as being limited by the parameter

$$
Z=\frac{R e}{\sqrt{W e}}=\frac{1}{O h}=\frac{\sqrt{\rho \gamma l}}{\eta}
$$

with

$$
1<Z<10
$$

where $O h$ is the Ohnesorge number, $l$ is a characteristic length, here taken to be the radius of the printing aperture, and $\rho, \gamma, \eta$ are respectively the density, surface tension and dynamic viscosity of the fluid. The limits defined in equation $1 \mathrm{~b}$ have been confirmed by experimental studies from a range of materials. ${ }^{5,7}$ The value of Oh has also been found to be a controlling factor in the volume and velocity of drops ejected during inkjet printing. ${ }^{8}$

Another aspect of the fluid that must be considered is its interaction with the substrate. The conventional application of inkjet printing is in text and graphics reproduction. In this case an image is formed through the deposition of isolated drops (or pixels) on a substrate. However, in order to form 2-dimensional (2-D) or 3dimensional (3-D) structures, printed droplets must coalesce to form liquid beads and planes. This is an important distinction between the use of inkjet printing as a manufacturing tool and its traditional application. 
It is already well established that an isolated liquid drop printed onto a flat surface will eventually spread to a shape defined by the equilibrium of surface forces and conventionally characterized by the equilibrium contact angle, $\theta_{\text {eqm }}$. Inkjet printed drops are sufficiently small, with diameter $<100 \mu \mathrm{m}$, that the shape of the surface is completely determined by capillarity and can be approximated to a spherical cap. A key behaviour in the fabrication of structures by inkjet printing is the interaction of these equilibrium sessile droplets on a substrate to form stable liquid beads or lines and the coalescence of these 1-D features to form more complex 2-D patterns. 3-D structures are produced by overprinting sequential layers and can be considered as the fabrication of a second 2-D pattern on a newly formed substrate layer. Hence we must consider the stability of liquid drops on a surface.

There has been considerable investigation of the interaction of liquid drops and the stability of liquid lines or beads prior to solidification by solvent loss through evaporation. If the liquid line is pinned at the contact line through evaporation and subsequent solute deposition, then, following the analysis of Davis, a parallel sided uniform section bead is stable. ${ }^{9}$ In which case, a series of regularly spaced individual drops positioned in a line can spread on a surface and interact to form a parallel sided liquid bead. It is readily seen that there must be a minimum drop spacing on the substrate to ensure that sequentially deposited drops spread across the surface and interact to form continuous features. Above this limit, simple principles of volume conservation can be used to predict the width of a stable liquid bead, $w$, formed by the overlap of spherical droplets of diameter $d_{0}$ and spacing on the substrate $p$, with ${ }^{10}$ 


$$
w=\sqrt{\frac{2 \pi d_{0}^{3}}{3 p\left(\frac{\theta}{\sin ^{2} \theta}-\frac{\cos \theta}{\sin \theta}\right)}}
$$

However there is also an upper limit to the width that can formed by the overlap of drops. When the drop spacing is sufficiently small a further instability occurs and the liquid bead contains regularly spaced bulges and does not show a uniform cross section. Duineveld showed that this bulging is a dynamic instability that depends on both the fluid substrate interaction and the rate at which drops arrive on the surface. ${ }^{11}$ Thus there is only a finite range of stable liquid beads, and hence solidified track widths, which can be fabricated by inkjet printing. ${ }^{12}$

Finally, after printing the fluid must undergo a phase change to produce a biomaterial of adequate mechanical properties. This phase transition should be isometric, ie should preserve volume and aspect of the liquid drops, and minimally exothermic to reduce the damage to neighbouring or encapsulated cells.

Because of the constraints listed above, specifically those on viscosity and thermicity, most inkjet printing studies targeting the preparation of biomaterials in the presence of cells have used physically gelling precursors. The most common precursor is sodium alginate, which undergoes a sol-gel transition when exposed to $\mathrm{Ca}^{2+}$ ions, thus becoming a structural material; ${ }^{2,13}$ also thermally-gelling polymers, such poly(N-isopropylacrylamide) (pNIPAm) have been used. ${ }^{14}$ However, physical gels lack the required robustness and stability for a practical tissue scaffold. The reversibility of the gelling process and the limited number of cross-links results in the gel structures possessing low moduli and being susceptible to dissolution in response 
to a number of environmental parameters, e.g. dilution, presence of calcium sequestrants, temperature, etc. Inter alia, their intrinsically reversible structure makes it difficult to render them selectively responsive to cell-mediated degradation.

As an alternative to this accepted route of physical gelation, we propose to use a dual gelling system that incorporates both physical and chemical gelation mechanisms ('tandem' gelation). ${ }^{15}$ A physical gelation mechanism is used to ensure a rapid phase change to occur on printing, thus allowing the fabrication of objects without issues of the stability of liquid beads and layers. This is followed by a secondary chemical crosslinking to increase the stability and the mechanical properties of the gelled deposit; because of the initial physical gelation, this can be undertaken as a secondary process after the printing of a complete layer of material. This combination of cross-linkable polymers and hydrogels will provide a robust tissue engineering solution to the manufacture of tissue scaffolds and their co-seeding with cells. Specifically, we use photopolymerization reactions for this purpose, because of a double advantage: a) easy control of the process because the crosslinking reaction does not depend on any other variable, such as mixing time of reagents, and can be switched on at any time in the process; b) by using appropriate masks, the resolution attainable with the process can be improved and further morphological features can be added to inkjet printed scaffolds.

There has been some preliminary work that demonstrates the concept as practical. The concept of 'tandem' gelation has already been employed in cell encapsulation, using Michael-type addition as a cross-linking reaction. Photopolymerization alone has been shown to be possible in the presence of cells: for 
example, mammalian cells have been successfully encapsulated in photopolymerisable PEG hydrogels using a free radical photoinitiator system of proven biocompatibility, such as Eosin Y/triethanolamine. ${ }^{16}$ This system has the advantage of using optical spectrum radiation to initiate cross-linking and thus minimising potential damage to cells from ionizing radiation. However, there have also been studies that demonstrate that other photoinitiators that operate at higher frequencies within the soft UV spectrum are also cytocompatible. ${ }^{17}$ Thus we believe that it is possible to develop a photopolymerisable cross-linked gel, for inkjet delivery, that will be cytocompatible and usable in cell scaffold applications. In this publication we report on such a material, its characteristics when used for inkjet delivery and the seeding of structures by cells also deposited using inkjet printing.

\section{Results and Discussion}

\section{Fluid Properties and Printing Conditions}

Pluronic F127, poly(ethylene glycol-bl-propylene glycol-bl-ethylene glycol) (PEOPPO), is known to show thermoreversible behavior in aqueous solution with a gel transition close to physiological temperatures. Figure 1a shows the viscosity of Pluronic F127 aqueous solutions as a function of temperature. There is a sharp increase in solution viscosity on increasing temperature above a transition in the region $10-20{ }^{\circ} \mathrm{C}$, depending on concentration. From our previous experience and manufacturer's data, the maximum viscosity for printing fluids through our printheads is approximately $30 \mathrm{mPas}$. Therefore we selected a $20 \mathrm{wt} \%$ solution for our studies. In order to allow both physical and chemical gelation, the PPO-PEO backbone of the Pluronic was functionalized with acrylate groups at each end (F127 DA); the 
photopolymerization of F127DA alone does not yield chemically cross-linked gels, due to the occurrence of intramicellar cross-linking (a detailed study of this phenomenon is the subject of a forthcoming publication), therefore a $10 \% \mathrm{wt}$. of poly(ethylene glycol) diacrylate (PEGDA, MW=575) was added to allow macroscopic gelation; the addition of PEGDA to allow the covalent cross-linking of F127DA micelles has already been used previously and was also employed for the preparation of temperature-sensitive cross-linked F127DA nanoparticles. ${ }^{17}$

From figure $1 \mathrm{~b}$ we can see that this functionalizing has little effect on the physical gelation properties of the Pluronic. The same is also true for the addition of the photoinitiators (Eosin Y/triethanolamine). The final set of data in figure 1b shows the gel transition for a solution of functionalized Pluronic F127 DA and PEGDA, which is the composition selected for subsequent printing trials. At the intended printing temperature of $5{ }^{\circ} \mathrm{C}$, the fluid viscosity is approximately $20 \mathrm{mPas}$. The surface tension of the F127DA / PEGDA solution was measured as $0.038 \mathrm{Nm}^{-1}$. We assume that the density of the printing solution is approximately $1000 \mathrm{kgm}^{-3}$ and that the characteristic length in equation 1 is given by the radius of the printer nozzle $\left(3 \times 10^{-5} \mathrm{~m}\right)$. Inserting this data into equation 1 a gives $R e / \sqrt{ } W e=1.7$; hence we conclude that the solution is thus within the printable range indicated in equation $1 b .^{4-6}$ In order to exploit the reversible thermal gelation properties of the system, inkjet printing was carried out with the printhead cooled to approximately $5{ }^{\circ} \mathrm{C}$ onto a substrate maintained at room temperature $\left(20-23{ }^{\circ} \mathrm{C}\right)$.

The contact angle of the printed solutions was characterized on two surfaces. Clean glass and glass functionalised with acrylate groups to improve adhesion of 
cross-linked gels. On a clean glass surface the mean contact angle from 8 measurements was $19^{\circ}$ and on the acrylate functionalized surface the contact angle was $53^{\circ}$.

\section{Printed Hydrogel Structures}

The drops printed using the F127DA / PEGDA solution had a mean diameter of $78 \mu \mathrm{m}$ in flight. Figure 2 shows an optical micrograph of printed hydrogel tracks on an acrylate functionalized glass surface produced by the overlap of a train of uniformly spaced printed drops. The width varied slightly alomg the track with $w=117 \pm 5 \mu \mathrm{m}$; tracks printed with a lateral separation $<100 \mu \mathrm{m}$ are seen to merge into a single wider track. If we insert the contact angle of the acrylate-displaying silanized glass into equation 2 , we can see that drops of mean diameter $78 \mu \mathrm{m}$, spaced $100 \mu \mathrm{m}$ are predicted to form a track of stable width $119 \mu \mathrm{m}$, showing very good agreement with the measured value. The two tracks that are printed with a separation smaller than the track width are seen to have merged with no identifiable features indicating their separation.

Figure 3 is an image obtained using an interferometer microscope and displays quantified height data as the contrast. a smooth transition in height, indicating that the coalescence of parallel tracks leads to a stable 2-D feature. Both the tracks and the merged tracks show a maximum height of about $5 \mu \mathrm{m}$ along the centre line. It is possible to estimate the height, $h$, of a liquid bead immediately after printing by assuming that it has a section of constant radius, ${ }^{9}$ in which case: 


$$
h=\frac{w\left(1-\cos \theta_{e q m}\right)}{2 \sin \theta_{\text {eqm }}}
$$

After inserting the measured line width of $117 \mu \mathrm{m}$ and a contact angle of $53^{\circ}$ into equation 3, the liquid bead height is predicted to be $29 \mu \mathrm{m}$. This is substantially greater than the height of a single track measured in figure 3; indicating that substantial volume loss that has occurred during film drying. We note that the tracks imaged in both figures 2 and 3 display a smooth profile with no evidence for segregation to the edges or "coffee staining" during drying and the large loss of solvent implied by the reduction in track height. Coffee staining is an ubiquitous problem in the inkjet printing of polymer solutions and is driven by fluid flows induced by differential solute evaporation across a liquid drop. ${ }^{19}$ However, coffee staining is known to be suppressed if the droplet solidifies or gels rapidly on impact ${ }^{20}$ and this local solidification is believed to explain the behaviour observed here.

The printed hydrogels were all characterized by the presence of a "shark skin" surface morphology (Figure 4), which was possibly due to a combination of drying and shrinkage phenomena during the time of irradiation. On rehydration and immersion in water, this defect disappears only to reappear when drying. Although this feature is clearly evident in figures 2 and 3, we believe that these are artefacts of the dry images we present and will not be present when the structures are used immersed in aqueous media during the culture of cells.

In figure 5 we explore the influence of the substrate surface energy and equilibrium contact angle on the printed tracks. In this image the track passes from a 
region of the glass surface that has been functionalised to a clean glass surface, the transition is clearly marked by an abrupt change in track width from $117 \pm 5 \mu \mathrm{m}$ on the functionalized surface to $240 \pm 10 \mu \mathrm{m}$ on unfunctionalized glass. The contact angle on the unfunctionalized glass surface was $19^{\circ}$; using this value in equation 3 returns a predicted liquid bead width of $210 \mu \mathrm{m}$, which is in reasonable agreement with the observed value, although not as good an agreement as was found on the, higher contact angle, functionalized glass surface. It is possible that the greater extent of droplet spreading required on the lower contact angle glass substrate may not be completed before the liquid is gelled by the increase in temperature generated by heat transfer from the substrate. Figure $5 \mathrm{~b}$ shows the printed tracks after immersion in water and rehydration. The gelled tracks on the unfunctionalized glass are not stable and are eroded or have been stripped from the substrate. In contrast the tracks on the acrylate functionalized glass show much greater stability because of the stronger chemical bond to the substrate.

In order to fabricate 3-D structures, it is necessary to overprint a series of layers on previously gelled material. Figure 6 shows the height of a printed track of as a function of the number of overprinted layers. The first layer has a thickness of slightly under $3 \mu \mathrm{m}$ (after drying) with subsequent layers increasing the thickness by increments of $4.5 \mu \mathrm{m}$, although the experimental scatter increases with increasing numbers of printed layers. We believe the thickness of the first printed layer in figure 6 to be anomalous as it is smaller than the heights we have determined from single printed tracks. The thickness of subsequent layers in the printed track height is consistent with our other measurements of single tracks. The increase in experimental 
scatter with increasing numbers of printed layers probably indicates a slight lack of register in the overprinting of sequential layers.

\section{Seeding Printed Structures with Cells}

The previous results have demonstrated the principle that photopolymerisable dual gelling materials can be inkjet printed to produce stable multilayer structures. This technology is now used to print simple cell containment structures. Figure 7 shows example structure fabricated for cell containment in the printed photocured and dried state (figure 7a and 7c) compared with the same structures after incubation in cell culture media for 12 hours (figures $7 b$ and $7 d$ ). We have fabricated a series of well arrays on silanized glass surfaces, defined as a crossed grid pattern with wall spacing $500 \mu \mathrm{m}$ in both the $x$ - and $y$-directions. In figure 7a and b a single layer of drops have been printed and the shark skin morphology is observed in the structure on immersion in cell culture media. We assume that this is in response to the considerable strains (and stresses) induced by the dehydration and rehydration of the gel. Such defects are no longer visible when bilayer or thicker structures are fabricated (figure $7 \mathrm{c}$ and $7 \mathrm{~d}$ ).

In order to produce structures to contain cells we printed a series of square wells of edge length $500 \mu \mathrm{m}$ from 4 overprinted layers and subsequently photo cured them. It is estimated that on rehydration, these structures will have a height $\approx 100 \mu \mathrm{m}$. A second printing cycle was then used to deposit human fibroblast cells into the structures. In figure 8 we can see that the drops have been successfully located within each of the square wells and that the printed walls of each well have contained the drops. In figure 9 we show cells printed into the wells after addition of further growth 
media and incubation; the acrylate functionalization of the glass surface results in a substrate that is not very adhesive to cells and incubation beyond 48 hours was required to allow the cells to fully attach to the substrate. However, it was relatively easy to promote cell adhesion by further treatment of the functionalized glass with either cysteamine or albumin, in either case cell adhesion was promoted within 24 hours incubation.

\section{Experimental}

\section{Materials}

Pluronic (poly(ethylene glycol-bl-propylene glycol-bl-ethylene glycol)) F127 (or Lutrol F127 or Poloxamer 407) with a MW in the range of 9840 to $14600 \mathrm{gmol}^{-1}$ and $=12,600 \mathrm{gmol}^{-1}$, containing a PEG content of $70 \% \mathrm{w} / \mathrm{w}$ was purchased from BASF (Ludwigshafen, Germany) or Sigma Aldrich (Gillingham, Dorset, UK) and used as received. Triethanolamine and poly(ethylene glycol) diacrylate with $=575 \mathrm{gmol}^{-1}$ were purchased from Sigma Aldrich (Buchs, Switzerland), eosin Y from Fluka (Buchs, Switzerland) and used as received.

\section{Preparation of glass substrates}

Glass microscope slides were functionalised with acrylate groups using the following procedure. 3-(trimethoxysilyl)propyl methacrylate was purchased from SigmaAldrich. Hexane, sulphuric acid, hydrogen peroxide, acetone, ethanol and glacial acetic acid were purchased from Fisher Scientific (Loughborough, UK). The glass slides are immersed in hexane for two hours to allow the complete removal of any residual 
coatings from glass manufacture; the slides are then rinsed with distilled water. In a second step, the glass slides are immersed in a 70:30 sulphuric acid:hydrogen peroxide (v/v) solution (piranha solution), stirring for $3-4$ hours or left overnight without stirring; followed by first rinsing with distilled water and then with acetone, before being left to dry. The reaction described here is from a modification of the protocol reported by Garoff and Ansorge. ${ }^{21} 0.51$ of distilled water was adjusted to $\mathrm{pH}$ 3.5 with glacial acetic acid, then $2 \mathrm{ml}$ of 3-(trimethoxysilyl)propyl methacrylate were added and the solution was stirred until clear; the glass slides were immersed in the solution for 1 hour while stirring, then they were washed thoroughly with distilled water first and with acetone later and left to dry. In a variation to this protocol, the reaction was carried out overnight without stirring the solution.

\section{Physical Characterisation}

Two rheometers were used to characterize solution viscosity as a function of shear rate and temperature: LVDV-III+ (Brookfield, Middleboro, MA, USA) with controlled temperature Bath/Circulator TC-501, fitted with a SC4-27 spindle (coneplate geometry), and a temperature-controlled Gemini Advanced Rheometer (Bohlin, Cirencester, UK), with parallel plates geometry (diameter of the plate $=20 \mathrm{~mm}$ ). The Brookfield rheometer required a sample volume of $13 \mathrm{ml}$. This was placed in the reservoir, located inside a thermostatic jacket and covered with a lid, to prevent water evaporation. Tests were performed applying a shear rate of $50 \mathrm{~s}^{-1}$ over a temperature range of $0-25^{\circ} \mathrm{C}$. The Bohlin rheometer required $50 \mu$ l of sample. The gap height between the plates was fixed at $100 \mu \mathrm{m}$ and the edge of the plate was surrounded with a dodecane solution, to prevent water evaporation from the sample. Tests were 
performed applying a shear rate of $10 \mathrm{~s}^{-1}$ and a temperature range of $5-40^{\circ} \mathrm{C}$ with an increase of temperature of $1^{\circ} \mathrm{C}$ per minute.

A Drop Shape Analysis System DSA100 (KRÜSS GmbH, Hamburg, Germany) was used to determine fluid surface tension using the pendant drop method. These measurements were carried out at room temperature $\left(23^{\circ} \mathrm{C}\right)$. The same equipment was used to determine the contact angles of drops on substrate surfaces but now using a sessile drop configuration.

\section{Inkjet printing and photopolymerization}

Inkjet printing experiments were carried out using the MPP-1000 printing platform; designed and constructed by the School of Materials, University of Manchester. This uses drop-on-demand (DOD) printing technology with piezoelectric actuated MJATP-01 printheads (Microfab Technologies, Plano, TX, USA) driven by a JetDrive III controller (Microfab) that provides a simple unipolar square wave activation pulse. The substrate is mounted on a motion table with controlled translation along the $x$ and $y$-axes (Micromech Systems, Braintree, UK) and the printhead is mounted on a rail to allow motion in the $z$-axis (Micromech). These are controlled using a Motion Perfect 2 Controller and software (Micromech). Positional accuracy of approximately $5 \mu \mathrm{m}$ is achievable with the system. The system is controlled through LabView 7.1. software (National Instruments, Austin, TX, USA) and this was used to define the waveform that activates the printhead (voltage, frequency, rise time, dwell time, falltime). We maintained the polymer solution and printhead at $5{ }^{\circ} \mathrm{C}$ and used the following parameters for the actuation signal, which was a unipolar square pulse of 
$100 \mathrm{~V}$ amplitude at $2500 \mathrm{~Hz}$ with rise, dwell, fall and echo times respectively of 3, 20, 3 and $3 \mu \mathrm{s}$. We set the distance between the printhead nozzle tip and the substrate to 2 $\mathrm{mm}$. Images of the ejection of drops were obtained using a CCD camera and a high brightness light emitting diode (LED) that was triggered by the JetDrive III controller to provide stroboscopic illumination. The droplets had a mean diameter of $78 \mu \mathrm{m}$ when in flight and were printed on the substrate with a spacing of $100 \mu \mathrm{m}$. After printing the gels were cross-linked by irradiating the samples with visible light using a L. E. Dementron I light source (SDS Kerr, Sybron Dental Specialties, Danbury, CT, USA), with wavelength $460 \pm 10 \mathrm{~nm}$.

The dimensions of printed structures were measured either using conventional optical microscopy or with a white light interferometric system (MicroXAM Surface Mapping Microscope, Phase Shift Technology Inc., Tucson, AZ, USA).

\section{Cell culture and cell printing}

The conditions used for printing cells were as reported in our earlier studies.3 Human fibrosarcoma (HT1080 fibrosarcoma) were obtained from the Faculty of Life Sciences, The University of Manchester. HT1080 fibroblast cells were cultured in DMEM (Dulbecco's modified Eagles' medium) containing $4.5 \mathrm{gl}^{-1}$ glucose, Glutamax TM, $10 \%$ foetal bovine serum (FBS), $1 \%$ Penicillin \& Streptomycin. The chondrocyte cells were cultured in DMEM containing: $1000 \mathrm{mgl}^{-1}$ glucose, Glutamax TM, 10\% FBS, $1 \%$ Penicillin \& Streptomycin and $50 \mathrm{mgl}^{-1}$ ascorbic acid V, all purchased from Invitrogen (Paisley, UK). Bovine Serum Albumin (BSA), Tris (2-carboxyethyl) phosphine hydrochloride (TCEP), sodium hydroxide standard solution $1 \mathrm{M}$, glycine 
and boric acid were purchased from Sigma Aldrich. Cysteamine was purchased from Fluka. Acetone was purchased from Fisher Scientific.

Cell suspensions for printing were prepared upon re-suspension of the cells, using the following procedure. After the media was removed from the culture flask, cells were rinsed with $10 \mathrm{ml}$ PBS, which was then discarded. The cells which attached to the flask surface were removed by the addition of $1 \times$ Trypsin EDTA. The flask was incubated at $37^{\circ} \mathrm{C}$ with $5 \% \mathrm{CO}_{2}$ (Heraeus ${ }^{\circledR}$ HERAcell® 150 incubator: DJB Labcare, Newport Pagnell, UK) and checked at regular intervals until cells assumed a round appearance (typically after 2 - 3 minutes), then they were transferred into a centrifuge tube and $3 \mathrm{ml}$ of fresh media were added and centrifuged at $1500 \mathrm{rpm}$ for 5 minutes. The spun cells formed a pellet and the supernatant liquid was removed and replaced with the required quantity of fresh media. To ensure uniform distribution and to disrupt cell clumps the final solution was gently agitated using a pipette (Fisher Scientific). The cell concentrations of the printing solutions were calculated using a haemocytometer (Fisher Scientific).

Intracellular fluorescent dye CFSE (Carboxy Fluorescein Succinimidyl Ester) was used to better locate cells in the scaffold after printing. Cells were stained with the dye using the following protocol: $1 \mu$ of diluted dye (1:10 DMSO) per $1 \mathrm{ml}$ of cells re-suspended in DPBS/5\% FBS. The cells are held for 5 minutes at room temperature and in the dark. This is followed by washing the cells twice by centrifuging and finally re-suspending them with DPBS/5\% FBS. 


\section{Conclusions}

We have successfully developed an acrylate difunctionalized triblock PPOPEO oligomer (Pluronic F127) that shows dual action gelling in a formulation that is suitable for inkjet printing. The shape and dimensions of printed tracks using this solution can be predicted using models developed for the printing of nanoparticle inks. 3-D structures can be generated through overprinting, and the height of resulting structures increases linearly with the number of layers printed. This system has been used to print simple square wells through overprinting and these wells have subsequently been seeded with cells and growth media through a second inkjet printing sequence.

The physical gelling used to initially fix the inkjet printed material prior to photocuring prevents material segregation and coffee staining despite significant shrinkage occurring from water loss. This shrinkage leads to a roughening of the dried hydrogel surface but this is removed on rehydration and immersion. Further work is needed to improve the adhesion of the printed well structures onto glass slides in order to provide surfaces that are adhesive to both the printed well material and cells.

\section{Acknowledgements}

This work was supported by the Engineering and Physical Sciences Research Council (EPSRC) through project grant EP/C004922/1. We would like to thank Jonathan Stringer and Andrew Wallwork for assistance with the inkjet printing experiments. 


\section{Notes and references}

1 T. Xu, J. Jin, C. Gregory, J.J. Hickman and T. Boland, Biomaterials, 2005, 26, 93.

2. M. Nakamura, A. Kobayashi, F. Takagi, A. Watanabe, Y. Hiruma, K. Ohuchi, Y. Iwasaki, M. Horie, I. Morita I and S. Takatani, Tissue Eng. 2005, 11, 1658.

3. R.E. Saunders, J.E. Gough and B. Derby, Biomaterials 2008, 29, 193.

4. B. Derby, Ann. Rev. Mater. Res. 2010, 40, 395.

5. B. Derby and N. Reis, MRS Bull. 2003, 28, 815 .

6. J.E. Fromm, 1984. IBM J. Res. Dev. 1984, 28, 322; N. Reis and B. Derby, 2000. in Materials Development for Direct Write Technologies, Boston, MA, 2000, MRS Symp. Proc. 2000, 624, 65: Materials Res. Soc.

7. D. Jang, D. Kim and J. Moon, Langmuir, 2009, 25, 2629; B. Derby, to appear in J. Europ. Ceram. Soc.

8. N. Reis, C. Ainsley and B. Derby, J. Appl. Phys. 2005,. 97, 094903.

9. S.H. Davis, J Fluid Mech. 1980, 98, 225.

10. P, Smith, D.Y. Shin, J. Stringer, B. Derby and N. Reis, J. Mater. Sci. 2006, 41, 4153.

11. P.C. Duineveld, J. Fluid Mech. 2003, 477, 175.

12. D. Soltman and V. Subramanian, Langmuir 2008, 24, 2224; J. Stringer and B. Derby, Langmuir 2010, 26, 10365.

13. T. Boland, X. Tao, B.J. Damon, B. Manley, P. Kesari, S. Jalota and S. Bhaduri, Mater. Sci. Eng. C 2007, 27, 372.

14. T. Boland, V. Mironov, A. Gutowska, E.A.Roth and R.R. Marwald, Anat. Rec. A, 2003, 272A, 497. 
15. F. Cellesi, N. Tirelli and J.A. Hubbell, Biomaterials, 2004, 25, 5115; F. Cellesi, W. Weber, M. Fussenegger, J.A. Hubbell and N. Tirelli, Biotechnol. Bioeng. $2004, \mathbf{8 8}, 740$.

16. G.M. Cruise, O.D. Hegre, D.S. Scharp and J.A. Hubbell, Biotechnol. Bioeng. 1998, 57, 655.

17. S.J. Bryant, C.R. Nuttelman and K.S. Anseth, J. Biomater. Sci. Poly. 2000, 11, 439.

18. D. Missirlis, N. Tirelli and. J.A. Hubbell, Langmuir, 2005, 21, 2605; D Missirlis, J.A. Hubbell and N. Tielli, Soft Matter, 2006, 2, 1067.

19. R.D. Deegan, O. Bakajin, T.F. Dupont, G. Huber, S.R. Nagel and T.A. Witten, Nature 1997, 389, 827.

20. P. Smith, B. Derby, N. Reis, A. Wallwork and C. Ainsley, In Euro Ceramics VIII, H. Mandal and L.Ovecoglu, Eds.; Trans Tech., Zurich, Switzerland, 2004, Vol. 1-3, p. 693; A/M. van den Berg, A.W.M. de Laat, P.J. Smith, J. Perelaer and U.S. Schubert, J. Mater. Chem. 2007, 17, 677.

21. H. Garoff and W. Ansorge, Annal. Biochem. 1981, 115, 450. 
a)

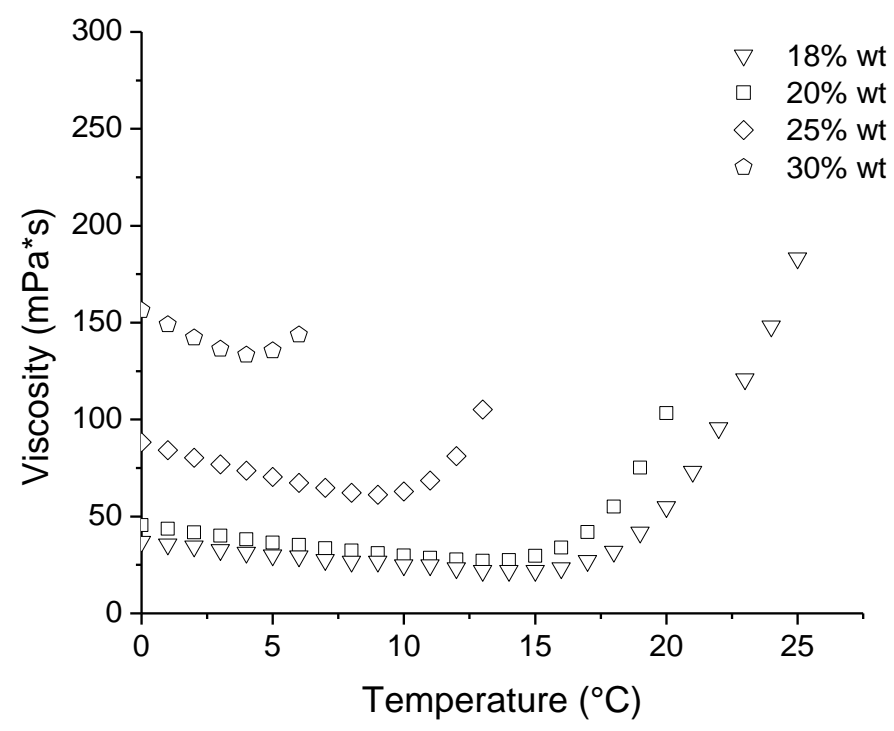

b)

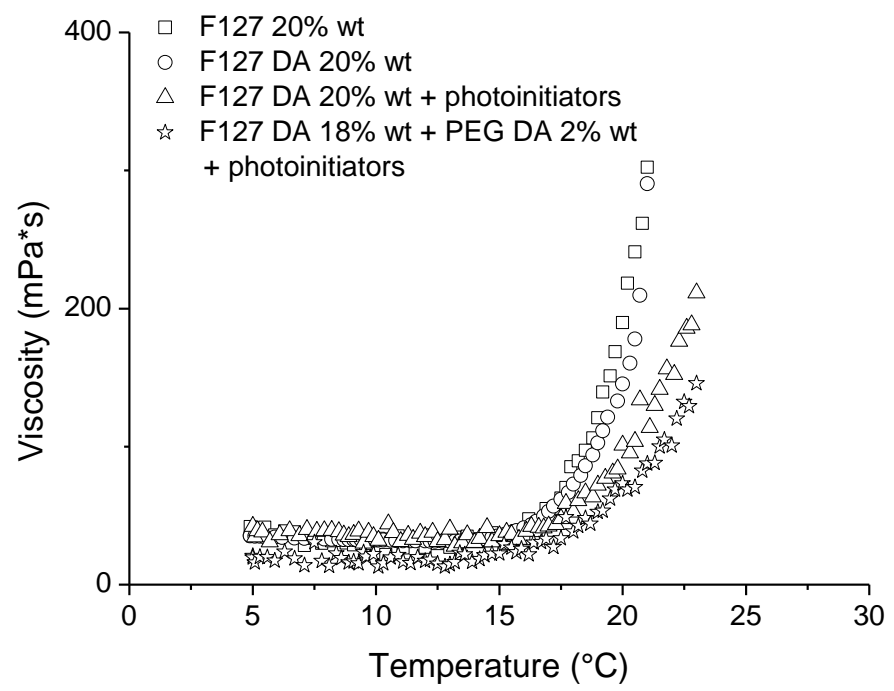

Figure 1 a) Viscosity of Pluronic F127 solutions as a function of temperature and concentration; b) Viscosity as a function of temperature for of solutions of Pluronic F127, F127 DA, F127 DA with photoinitiators and F127 DA with photoinitiators and PEG DA. 


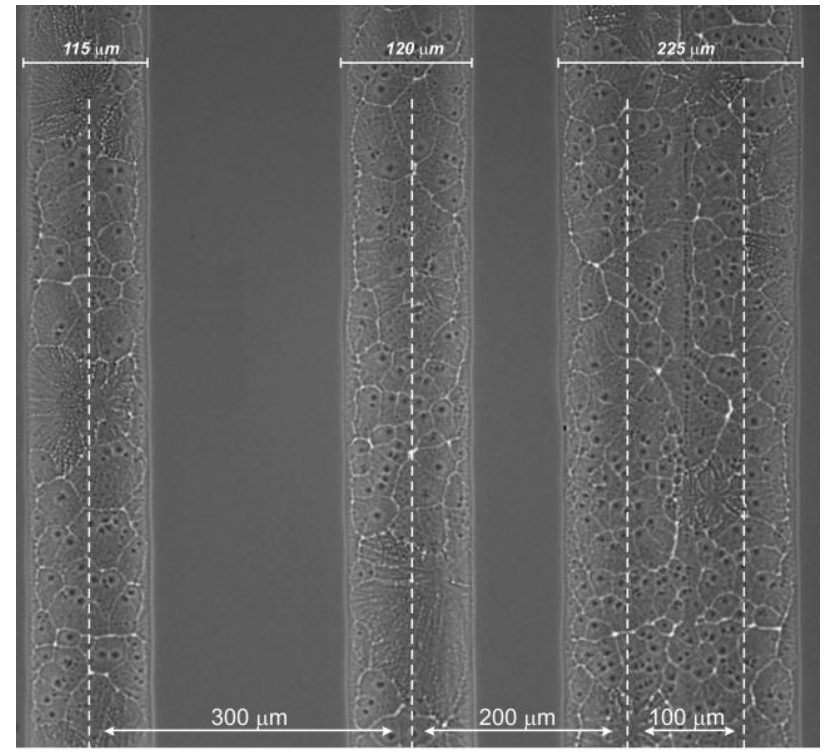

Figure 2 Bright field optical microscopy images showing parallel sided inkjet printed tracks after photo-crosslinking. If parallel tracks are printed with a separation $<100 \mu \mathrm{m}$ they blend together to form a 2-D structure. 


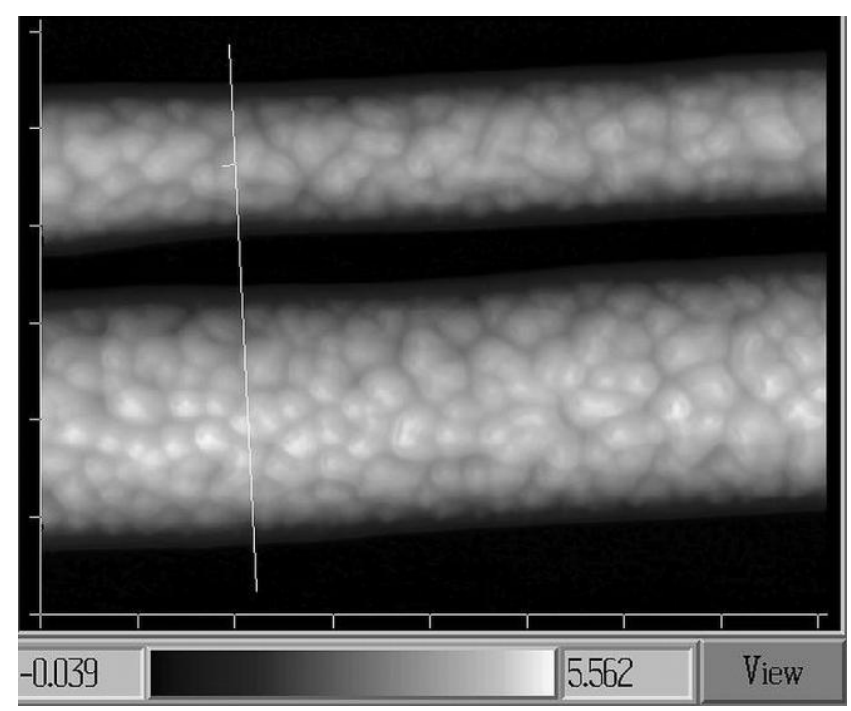

Figure 3 Phase contrast images of printed and photocrosslinked tracks, contrast indicates height. Upper track is made with a single printing pass with drop spacing $100 \mu \mathrm{m}$. Lower track is made from two overlapping passes of drop spacing 100 $\mu \mathrm{m}$, with a lateral separation of $100 \mu \mathrm{m}$. In both cases the tracks show a height maximum in the centre and no evidence of coffee staining at the edges. 


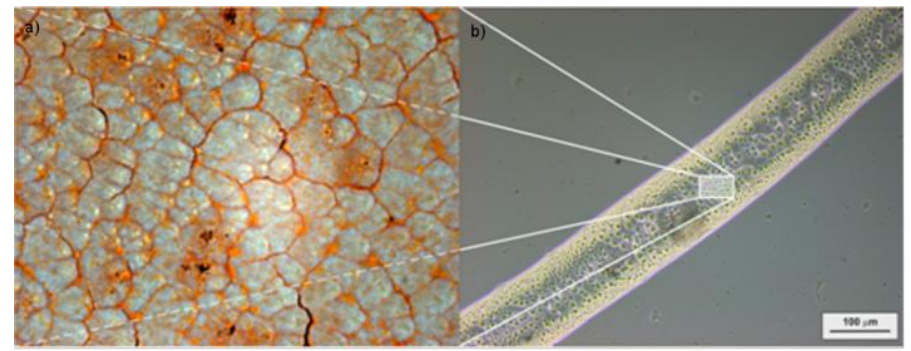

Figure 4 Inkjet printed tracks after photopolymerization (b) showed a domainstructured surface ("shark-skin"), with a magnified image in (a) showing interdomain regions characterized by the presence of residual initiator (orange coloured areas).

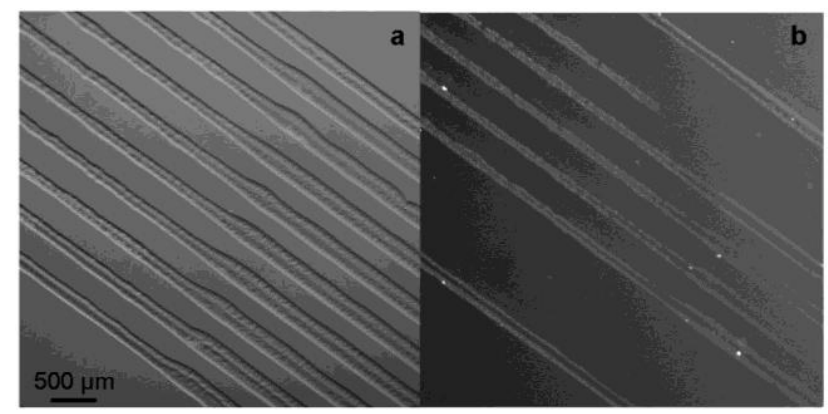

Figure 5 F127DA gel printed onto glass slide silanized along half of its total length. (a) structure after photopolymerisation, boundary between silanized (upper left) and unsilanized glass (lower right) is clearly visible showing a change in printed line width; (b) Structure after immersion in water, showing better retention of the printed structures on the silanized surface. 


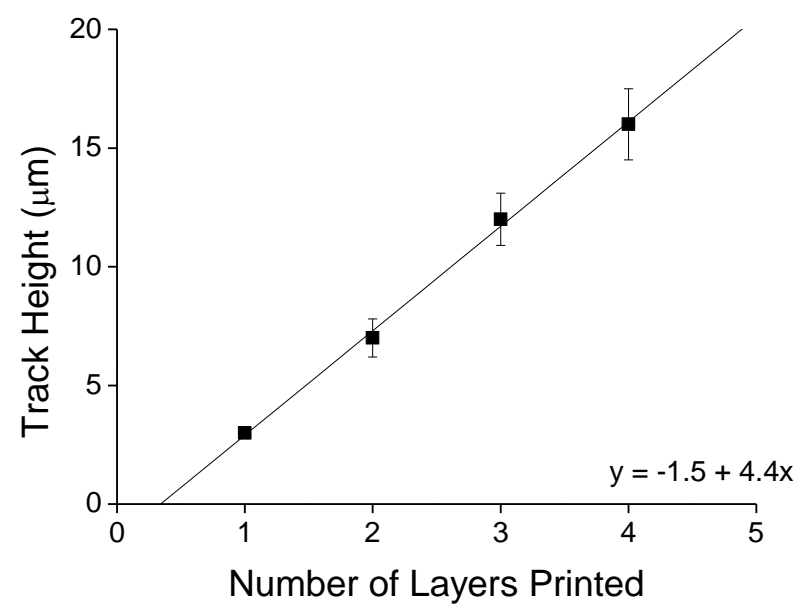

Figure 6. Height of a printed gel layer as a function of number of layers printed. There is a good linear relation between thickness and number of printing passes, although the experimental scatter also increases with the number of printed layers.

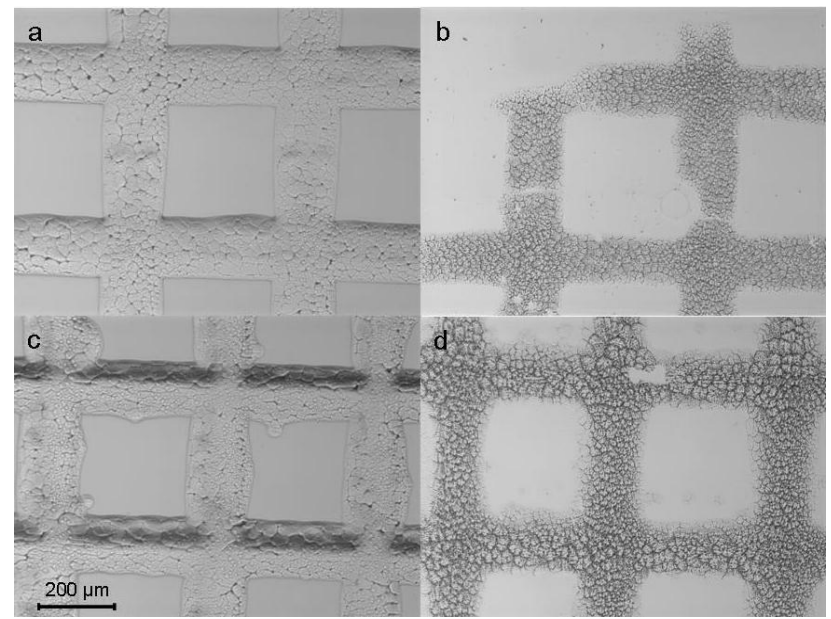

Figure 7 Multi-chamber pattern printed on a $500 \mu \mathrm{m}$ x $500 \mu \mathrm{m}$ grid. (a) single printed layer after photocrosslinking, (b) single layer structure incubated for 12 hours in cell culture media; (c) printed bilayer structure after photopolymerisation (d) bi-layer structures incubated for 12 hours in cell-culturing media. 


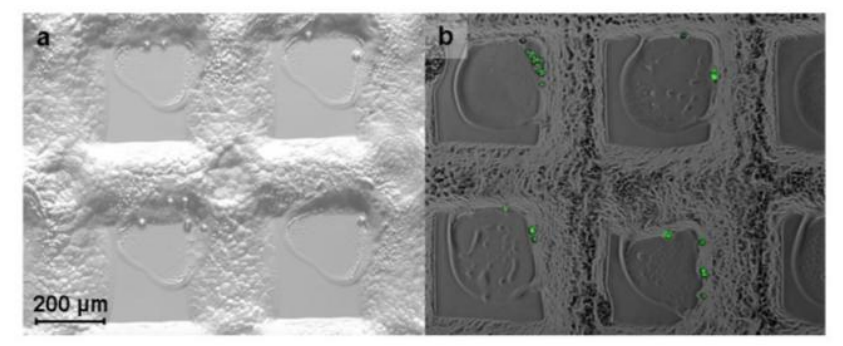

Figure 8 Multi-chamber pattern printed on a $500 \mu \mathrm{m}$ x $500 \mu \mathrm{m}$ grid, photocured and subsequently cell-seeded by inkjet printing, showing the presence of each printed droplet contained by the cell walls.

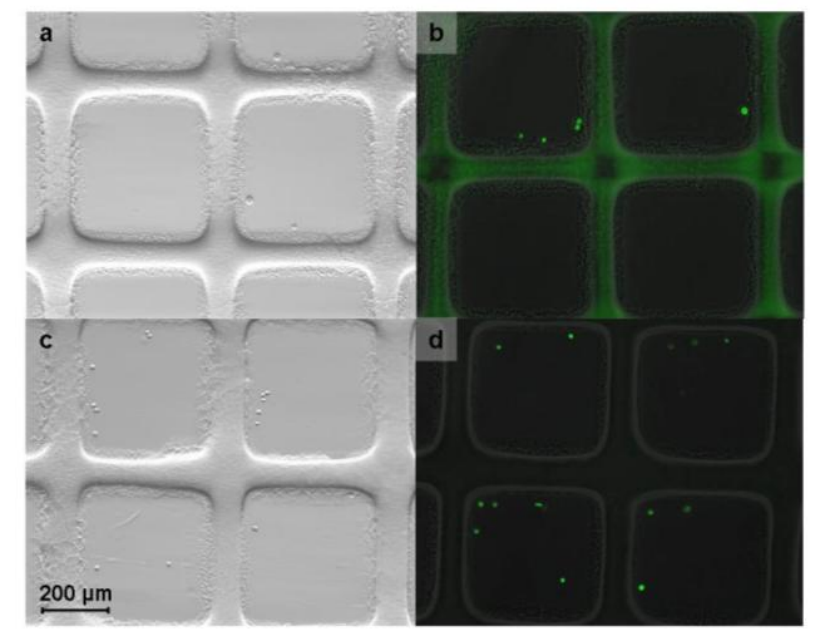

Figure 9 Images obtained from a multi-chamber pattern printed on a $500 \mu \mathrm{m} \mathrm{x}$ $500 \mu \mathrm{m}$ grid, photocured and cell seeded by inkjet printing after the provision of further media and incubation for 24 hours. Note that all trace of the shark skin surface relief has been eliminated by immersion in fluid and subsequent rehydration. 